\title{
Enhancement of Ion Transmission at Low Collision Energies via Modifications to the Interface Region of a Four-Sector Tandem Mass Spectrometer
}

\author{
Wen Yu and Stephen A. Martin \\ Genetics Institute, Andover, Massachusetts, USA
}

The transmission efficiency of precursor and product ions decreases significantly at lower collision energies in a four-sector tandem mass spectrometer. In an effort to improve the overall ion transmission in this energy regime three modifications were made in the interface region between the two stages of mass analysis. An einzel lens was inserted prior to the deceleration lens of the collision cell block to reduce the precursor ion beam diameter. The collision cell block was reduced in thickness while maintaining the collision path length, thus increasing the number of ions which entered and exited the gas chamber, while removing any stray electrical fields. Finally, a second active focusing element was incorporated after the collision cell block to enhance the collection efficiency of the product ions. $A$ tandem mass spectrum of angiotensin I obtained with this interface, at a collision cell block potential of 9200 volts, exhibited classical high energy collision-induced dissociation (CID) fragmentation patterns, a precursor ion transmission of $92 \%$ and an overall CID efficiency of approximately $7.5 \%$. These improvements have resulted in a dramatically higher overall ion transmission at high collision cell potentials as well as sufficient sensitivity in acquiring good quality CID spectra in the lower collision energy regime (i.e., $60 \mathrm{eV}$ ). (J $1 \mathrm{tm}$ Soc Mass Spectront $1994,5,460-469$ )

$\mathrm{T}$ landem mass spectrometry (MS/MS) has been widely utilized for determining the primary structure of peptides and other biolugical molecules, thus adding another dimension to the information obtained from the mass measurement of these molecules [1]. The ultimate goal of this technique is to achieve bond cleavage in the molecule via efficient energy transfer and obtain reproducible and predictable fragmentation patterns from which the peptide sequence may be determined. A variety of instruments and excitation processes have been applied to the structural characterization of peptides, each with a varying degree of success [2-4]. A number of these studies have used high energy collision-induced dissociation (CID) combined with tandem four-sector mass spectrometers [5-7]. This approach has a number of performance features including high resolution in both mass analyzers, accurate mass assignments, and reproducibility of the fragmentation patterns. These features, combined with the unique fragmentation pathways obtained via high energy CID [8, 9], make this an appropriate technique for solving structural problems,

Address reprint requests to Stephen A. Martin, Genetics Institute, One Burtt Road, Andover, MA 01810. particularly in situations where the protein is posttranslationally modified.

One objective of mass spectrometry is to provide as much information as possible about the structure of a molecule while utilizing a minimum amount of sample. This goal is a prerequisite if mass spectrometry is to contribute to the structural characterization of biologically significant compounds, which are frequently isolated in trace amounts. In these characterization strategies, molecular mass analysis is an initial step providing an overall view of the molecule. MS/MS is a subsequent step which may provide primary structural information. However, these analyses require more material than molecular mass measurements. As a technique, MS/MS may be divided into three processes: ion formation, fragmentation, and detection. Improvements in any of these areas will improve its overall sensitivity, thereby increasing the range of projects which might benefit from its inclusion in the overall characterization strategy.

There have been a number of improvements in the methods of ionization for peptides and proteins such as with fast-atom bombardment (FAB) [10], continuous-flow FAB (CF-FAB) [11], and more recently, electrospray ionization (ESI) [12] and matrix-assisted laser 
desorption ionization (MALDI) [13]. Each technique has further increased the sensitivity as well as opened up new areas of applications for mass spectrometry, independently of the type of analyzer. In the area of ion detection, recent developments on magnetic sector instruments have resulted in the implementation of array detectors which permit the simultaneous detection of up to $40 \%$ of the mass range [14]. These devices yield a factor of 50 to 100 increase in overall ion detection. These improvements in ion formation and detection have substantially reduced the amount of material required to determine the molecular mass and/or provide primary sequence information. However, neither of these improvements has had any effect on the relative percent of ions which are transmitted between the two mass analyzers or are fragmented in the interface region as a function of the collision cell potential. Experimentally, significantly more material is still required for a MS/MS measurement versus a molecular weight determination in our four-sector mass spectrometer.

We had noted previously ( $Y u, W$.; Martin, S. A. Manuscript in preparation) that precursor ions could have a higher apparent CID efficiency when they undergo collision at a lower kinetic energy (lab frame) (i.c., a higher collision cell potential) in our four-sector mass spectrometer. Furthermore, under the appropriate conditions, high energy CID fragmentation patterns of peptides were retained at these lower kinetic energies by using a heavier collision gas [15]. Unfortunately, the overall sensitivity or "spectrum quality" did not increase significantly, partially due to low ion transmission efficiency of the original MS/MS interface at these higher collision cell potentials. In this work, we present mudifications to the MS/MS interface of a JEOL HX110/HX110 tandem mass spectrometer, which results in higher ion transmission efficiency over a broad range of conditions and better collection efficiency of product ions.

The objective of this work was to evaluate the various parameters which may have an impact on the ion transmission through the interface region of our JEOL HX110/HX110 tandem mass spectrometer. Factors in this study which were considered to contribute to the overall efficiency include ion transmission (precursor and product), fragmentation efficiency, and collision cell potential. The goal was to improve transmission efficiency and thereby improve the sensitivity for the CID of peptides at high collision cell potentials.

\section{ExperimentaI}

\section{Instrument}

Original lnterface. The mass spectrometer used in this work is a JEOL HX110/HX110HF tandem double focusing instrument with EBEB geometry (Figure 1). The MS/MS interface is located between the detector slit of the first mass spectrometer (MS-1) and the source slit

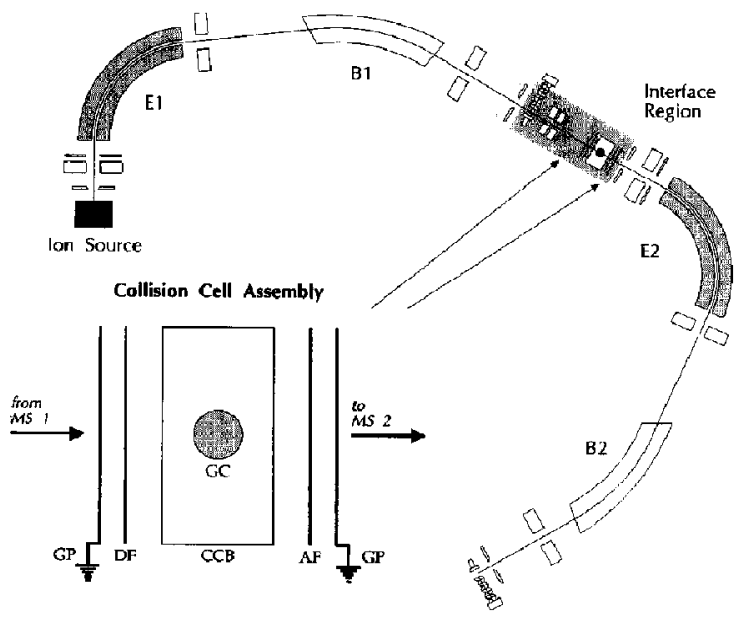

Figure 1. Schematic representation of the JEOL HX110/ $\mathrm{HX} 110 \mathrm{HF}$ tandem mass spectrometer with the standard interface region. An expanded view of the collision cell assembly is shown in the inset. This assembiy consists of GP, DF, restrictive entrance and exit apertures to the $\mathrm{CCB}, \mathrm{CC}$ region centered within the $C C B$ and $A F$.

of the second mass spectrometer (MS-2) as shown in Figure 1. The interface region is subdivided into three segments for the purpose of this study. The first segment contains two groups of quadrupole focusing lenses followed by a pair of $Y$ and $Z$ deflection lenses (Ishihara, M. JEOL private communication). The second segment is a field-free region, approximately 26 $\mathrm{cm}$ in length prior to the collision cell assembly, which is the third segment of the interface. The collision cell assembly of the original interface is shown in detail in the inset of Figure 1. It consists of a ground plate (GP), deceleration focusing lens (DF), collision cell block (CCB), gas cell (GC), acceleration focusing lens ( $A F)$, and a final ground plate (GP) prior to the entrance slit of the second mass spectrometer.

Modifications to Interface. As described in the previous section, the original MS/MS interface region consisted of three segments: focusing elements, field-free area, and the collision cell assembly. Three discrete modifications were made to this interface in the field-free and collision cell segments as shown in the comparison of the SIMION representation of the key elements (Figure 2). Because no mechanical modifications were made to the first segment of the interface region, consisting of the quadrupole lenses and the $Y$ and $Z$ deflectors, these elements are not shown in this figure.

The first modification involved the addition of an einzel lens assembly in the field-free segment of the interface region before the collision cell assembly (Figure 2b). The einzel lens was constructed of three $5 \mathrm{~mm}$ thick stainless steel rings with an inner diameter of $10.5 \mathrm{~mm}$. The lens elements are mounted in a grounded stainless steel tube which is slotted to allow for adjust- 
a

Field Free Region

Collisian Cell Assembly

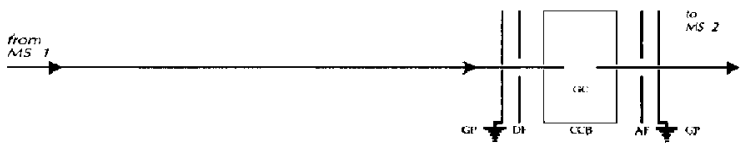

b

Einzel Lens Assembly Collision Cell Assembly

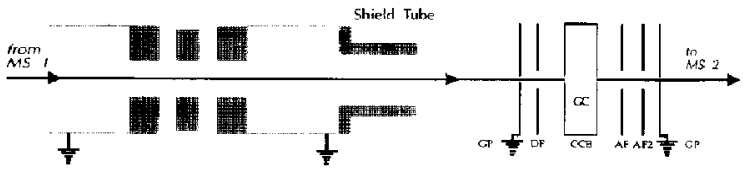

Figure 2. Comparison of (a) the original and (b) the modified MS/MS interface regions of the JEOL HX110/HX110 tandem mass spectrometer as viewed from the top of the interface. The modifications consist of (1) the addition of an EZ and ion shield tube ( $\mathrm{EZ}$ assembly) in the field-free region before the collision cell assembly, (2) a $70 \%$ reduction in the collision cell block width, (3) addition of a second acceleration and focusing lens (AF2) after the CCB, and (4) a change in the position of the first ground plate and the DF lens elements with respect to MS-1. No change was made to the collision gas path length or in the position of the second ground plate with respect to the entrance slit of $\mathrm{MS}-2$.

ment of the lens element positions or the addition of more elements. The active element of the einzel lens assembly is controlled by a separate power supply, variable from 0 to $5 \mathrm{kV}$, when the accelerating voltage of MS-1 is $10 \mathrm{kV}$.

The second modification involved replacing the original collision cell block with a stainless steel block in which the thickness, in the direction of the principal motion of the ions, was reduced from $40 \mathrm{~mm}$ to 12 mm. The effective ion-gas collision path length remain unchanged. In the modified collision cell block used for these studies, the gas is introduced from the top of the block, as opposed to the original collision cell block in which the gas is introduced via a port in the side of the cell. (Note: as a result of space limitations in the final design of the new collision cell flange assembly the gas is now introduced into the cell via a side port.) The entrance and exit slits of the gas cell are each defined by a removable plate which contains a $2 \times 6$ $\mathrm{mm}$ slit. All excess metal and mounting hardware were removed from the collision cell to avoid fringing fields which could perturb the trajectories of the precursor and/or product ions. Whenever practical, the high voltage leads to the lens elements and the collision cell block were also shielded from the ion path.

The third modification involved the addition of a second lens element, referred to as AF2, in the acceleration region after the ions had undergone dissociation in the collision cell. This lens is located the same distance from the first lens as the ground lens in the original cell design. The ground plate was added behind this lens and placed such that its distance from the source slit of MS-2 was the same as in the original setup. The relative positions of the first ground plate and the deceleration focusing lens elements with respect to the collision cell block are the same as in the original design; as a result, the distance from the exit slit of MS-1 to the first ground plate is longer in this design (Figure 2). The potential, variable from 0 to 5 $\mathrm{kV}$, for the second acceleration lens element is provided by a separate power supply. The additional power supplies, collision cell body and the einzel lens assembly were constructed by J. A. Hill Instruments, Inc. (Arlington, MA).

\section{Experimentally Measured Parameters}

Collision Energy. The ion beam kinetic energy was measured as the potential difference between the ion acceleration potential in MS-1, which is fixed at 10,000 $\mathrm{V}$ in these studies, and the collision cell potential which is variable from 0 to $10,000 \mathrm{~V}$. The precision of these measurements is limited by the hardware to \pm 10 $\mathrm{eV}$. In the event of collision with neutral gas, the ion kinetic energy at the point of collision is taken as the collision energy. The collision energy in lab frame $\left(E_{\mathrm{lab}}\right)$ is converted to the collision energy in the centerof-mass conordinates ( $F_{\text {sit }}$ ) with the formula:

$$
E_{\mathrm{cm}}=E_{\mathrm{lab}} * m_{\mathrm{gas}} /\left(M_{\mathrm{ion}}+m_{\mathrm{gas}}\right)
$$

where $M_{\text {ion }}$ and $m_{\text {gas }}$ are the mass of precursor ion and collision gas, respectively. The center-of-mass energy, $E_{\mathrm{cm}}$, represents the maximum kinetic energy available to be converted to internal energy of the precursor ions.

Transmission and Apparent CID Efficiency. The objective of this study was to characterize the various parameters in the interface region of the JEOL HX110/HX110 tandem mass spectrometer which affects the overall MS/MS sensitivity at high collision cell potentials, such as low collision energies. The efficiency of the MS/MS interface is represented by the following coefficients: precursor ion transmission efficiency, $\epsilon_{\text {trans; }}$ CID efficiency, $\epsilon_{\mathrm{CID}} ;$ and the collection efficiency, $\epsilon_{\text {collect }}$. Based on these definitions, equations are derived for the overall efficiency, $\epsilon_{\text {overall, }}$ and the apparent CID efficiency, $\epsilon_{\text {apparent }}$.

$$
\epsilon_{\text {overall }}=\epsilon_{\text {trans }} * \epsilon_{\text {apparepnt }}
$$

and

$$
\epsilon_{\text {apparent }}-\epsilon_{\text {CID }} * \epsilon_{\text {collect }}
$$

The precursor ion transmission efficiency, $\epsilon_{\text {trans }}$, may be defined as the percentage of ions detected after mass analysis in MS-1 and after the same ions have traveled through the interface region and the second mass spectrometer to the final detector in the absence 
of a collision gas using the formula:

$$
\epsilon_{\text {trans }}=I_{\mathrm{MS}-2} / I_{\mathrm{MS}-1}
$$

where $I_{\text {MS-1 }}$ and $I_{\mathrm{MS}-2}$ are the ion intensities measured at the MS-1 and MS-2 detectors, respectively. The precursor ion current at each of the detectors was determined using a signal-to-noise level method developed by Green which compensates for the variability in the response factor of the two detectors (Green, B. VG private communication). The precursor ion transmission efficiency at a collision cell block potential of 3000 volts was measured to be $100 \pm 5 \%$, based on the corrected ion current at MS-1 and MS-2.

$\epsilon_{\mathrm{CID}}$ is the fraction of the precursor ions which undergo collision and dissociate in the collision region (note $\boldsymbol{f}_{\mathrm{CID}}$ is related to several factors, i.e., collision energy, collision gas, pressure, etc.). $\epsilon_{\text {collect }}$ is the fraction of the product ions which reach the MS-2 detector. These two terms, $\epsilon_{\mathrm{CID}}$ and $\epsilon_{\text {collect }}$, carnot be independently measured in our current experimental setup; however, the collective effect of these terms, $\boldsymbol{E}_{\text {apparent }}$, may be measured as described below.

The apparent CID efficiency, $\epsilon_{\text {apparent }}$, is defined for singly charged precursor ions as

$$
\epsilon_{\text {apparent }}=\left[\left(\Sigma I_{\mathrm{d}}\right) /\left(I_{\mathrm{p}} *\left(1-T_{\mathrm{p}}\right) / T_{\mathrm{p}}\right)\right]
$$

where $I_{\mathrm{d}}$ is the intensity of the product ions, $I_{\mathrm{p}}$ is the intensity of the precursor ion signal after attenuation with a collision gas, and $T_{P}$ is the fraction of the precursor ion signal remaining after addition of the collision gas. The result of the denominator in eq $\mathbf{5}$ is the precursor ion intensity available for the CID process.

Since the precursor ion intensities are measured prior to and after attenuation with a collision gas at a given collision cell block potential, the overall precursor ion transmission efficiency is not a factor in the equation for $\epsilon_{\text {apparent. }}$. The percentage of the precursor ions which are transmitted through the interface as a function of the collision cell potential are accounted for in $\epsilon_{\text {overall }}$, eq 2. On the basis of this equation, the sensitivity of an MS/MS experiment is directly related to $\epsilon_{\text {overall }}$ and it may be improved by increasing the efficiency of any of the individual steps. This expression of efficiency differs from that defined by Yost et al. [16] in that it has a separate term for the precursor ion transmission and considers the difference in precursor ion intensity, prior to and after attenuation, as the total ion intensity available for the CID process.

Experimentally, the measurement of the apparent CID efficiency is obtained with a multistep process. First, the precursor ion is transmitted through the collision cell, which has been set to a potential based on the collision energy of the experiment, to the MS-2 detector. Collision gas is then introduced into the collision cell until the precursor ion signal, measured at the second detector, is reduced to approximately $25 \%$ of its original intensity $\left(T_{\mathrm{P}}\right)$. The intensity of this attenuated precursor ion signal at the MS-2 detector is adjusted so that its signal is not saturated. This step is required to obtain accurate peak areas for $I_{\mathrm{p}}$. Second, seven product ion spectra are obtained in series. The first and last are acquired with a $1 \times$ preamplifier gain setting on the detector so that accurate precursor ion intensities are obtained. The middle five product ion spectra are acquired with a $10 \times$ gain on the preamplifier. Five product ion spectra are acquired to insure good ion statistics. The $10 \times$ gain was chosen to increase the signal for these ions. The measurement of the precursor ion intensity, before and after these spectra, is used to correct for any decrease in total ion signal that is caused by depletion of the sample during these measurements. The areas of all the product ions in scans 2-6 are summed together, divided by 10 to correct for the gain, and then divided by the total number of scans. This value corresponds to $\Sigma I_{d}$ in eq 5. The average of the precursor ion intensity from scans 1 and 7 is $I_{\mathrm{P}}$.

\section{Reagents}

Angiotensin I (DRVYTHPFHL, [M $+\mathrm{H}]^{+}$1296.7, monoisotopic) was purchased from BACHEM (Lajolla, CA) and used without purification. About $0.3 \mu \mathrm{L}$ of the sample solution $(1-5 \mathrm{nmol} / \mu \mathrm{l})$ was placed on the probe tip together with $0.4 \mu \mathrm{L}$ of nitrobenzyl alcohol and $0.2 \mu \mathrm{L}$ of a $5 \%$ acetic acid solution. The singly charged ions were produced with a standard JEOL $\mathrm{Cs}^{+}$liquid secondary ionization mass spectrometry ion source with a filament current of $2.2 \mathrm{~A}$ and an ion gun potential of 15 to $22 \mathrm{kV}$.

\section{Results and Discussion}

\section{Transmission Efficiencies of the MS / MS Interface}

The difference between $\epsilon_{\text {overall }}$ and $\epsilon_{\text {apparent }}$ can be illustrated by the comparison of the CID mass spectra of angiotensin I at two collision energies $(8000 \mathrm{eV}$ and $800 \mathrm{eV}$ ) with the original MS/MS interface (Figure 3). At a collision energy of $8000 \mathrm{eV}$ with helium as the collision gas (Figure 3a) $\epsilon_{\text {apparent }}=2.4 \%$, using the protocol described in the Experimental section. As a comparison, $\epsilon_{\text {apparent }}=6.6 \%$ for the same CID spectrum taken at $800 \mathrm{eV}$ collision energy with xenon as the collision gas (Figure 3b). It should be noted that in the instrument used in this study helium yields higher $\epsilon_{\text {apparent }}$ values at higher collision energies $(>1000$ $\mathrm{eV}$ ), whereas, below these values xenon produces higher apparent efficiencies. Both these spectra exhibit high energy fragmentation patterns [15].

These data indicate that $\epsilon_{\text {apparent }}$ is higher at the lower collision energies ( $Y u, W$.; Martin, S. A. Manuscript in preparation). This suggests that better sensitivity for routine tandem mass spectra would be obtained at these lower collision energies. Unfortu- 

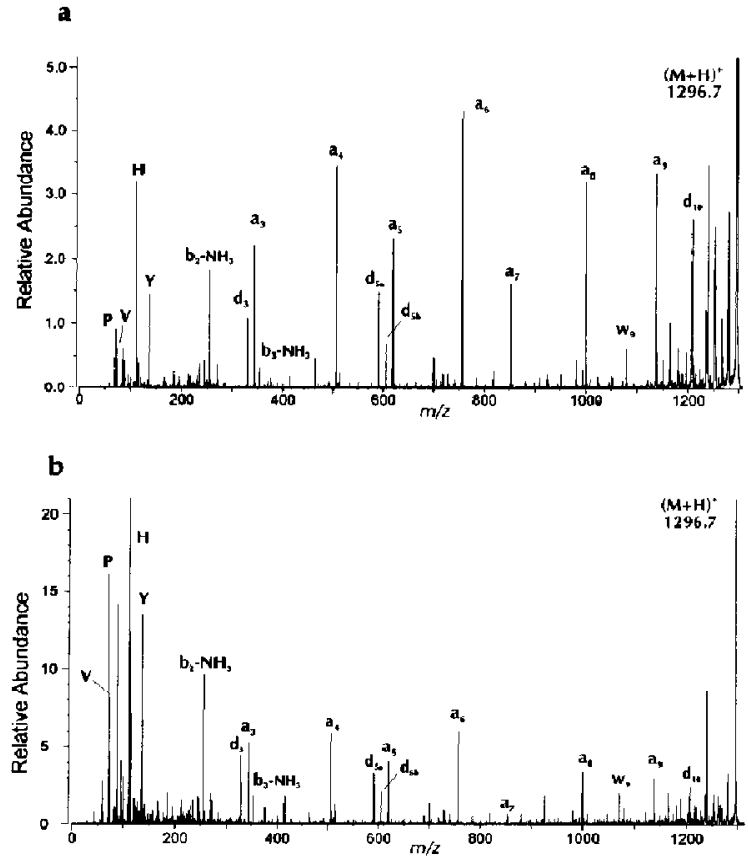

Figure 3. Comparison of the fragmentation patterns and apparent CID efficiency, $\epsilon_{\text {apparent, }}$ in the tandem mass spectra of angiotensin I (DRVYIHPFHL, $[\mathrm{M}+\mathrm{H}]^{+}$1296.7) as a function of collision energy with the original interface region. (a) Tandem mass spectrum of angiotensin 1 at a collision energy of $8000 \mathrm{eV}$ (lab) or $24.6 \mathrm{eV}$ (center-of-mass). The precursor ions were attenuated to $20 \%$ with helium as collision gas, $\epsilon_{\text {apparent }}=2.4 \%$. (b) Tandem mass spectrum of angiotensin $I$ at the collision energy of $800 \mathrm{eV}$ (lab) or $73.5 \mathrm{eV}$ (center-of-mass). The precursor ion was attenuated to $20 \%$ with xenon as collision gas, $\epsilon_{\text {ар parent }}=6.6 \%$.

nately, because the precursor ion transmission at this lower collision energy $(800 \mathrm{eV})$ is three- to fourfold lower than that at the higher collision energy (see below) there is no real enhancement, $\left(\epsilon_{\text {overall }}(8000 \mathrm{eV})\right.$ $\left.=2.4 \%, \epsilon_{\text {overall }}(800 \mathrm{eV})=2.2 \%\right)$. These results suggest that if $\epsilon_{\text {trans }}$ could be improved by a factor of three to four at these high collision cell potentials while $\epsilon_{\mathrm{CID}}$ and $\epsilon_{\text {collect }}$ were kept constant or improved, then $\epsilon_{\text {overall }}$ for angiolensin I would be significantly better at these lower collision energies.

\section{Modeling of the MS / MS Interface}

Original Collision Cell. Based on the experimental observation that the precursor ion transmission through the collision cell begins to decrease as a function of increasing the collision cell potential above $8000 \mathrm{~V}$ (Figure 4), the first step was to model the precursor ion trajectories over a range of collision cell potentials. This modeling was performed with the ion optical program, SIMION $[17,18]$. The original collision cell was represented in SIMION by defining a potential array, as shown in Figure 5. Electrodes representing the two shield GPs, a pair of DF and AF lens elements

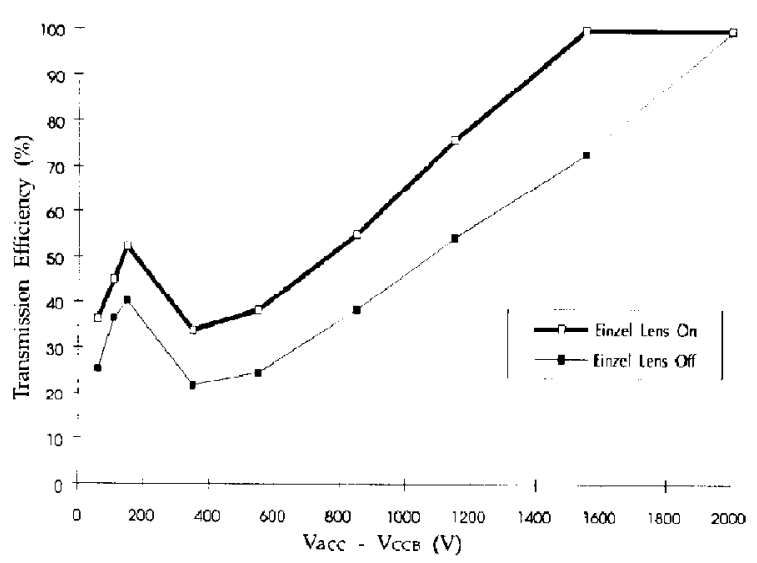

Figure 4. A comparison of the relative transmission efficiency $\left(\epsilon_{\text {trans }}\right)$ of precursor ions through the original MS/MS interface region of the JEOL $\mathrm{HX} 110 / \mathrm{HX} 110$ tandem mass spectrometer (EZ off) and one modified by the addition of an EZ in the field-free region as a function of collision energy $\left(V_{\text {acc }}-V_{\mathrm{CCB}}\right)$. $V_{\text {acc }}$ and $V_{\mathrm{CCB}}$ are the MS-1 acceleration and the CCB potentials, respectively. For the original MS/MS interface, the precursor ion transmission efficiency is better than $90 \%$ at $V_{\text {acc }}-V_{C C B}=2000 \mathrm{~V}$. Therefore, a reference collision cell potential of $8000 \mathrm{~V}$ is used for the calculation of $\varepsilon_{\text {trans }}$ in this figure.

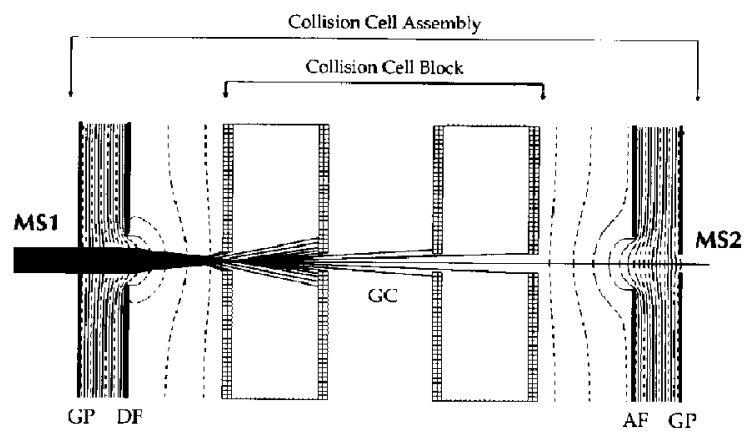

Figure 5. The SIMION model of the original collision cell assembly of the MS/MS interface. The electrode elements of the SIMION model and their potentials are: $G P=0 \mathrm{~V}, D F=8010 \mathrm{~V}$, $\mathrm{CCB}=9250 \mathrm{~V}, \mathrm{GC}=9250 \mathrm{~V}, \mathrm{AF}=7940 \mathrm{~V}$. All potentials are determined experimentally from the optimized interface. The ion trajectories are plotted for a precursor ion beam with an initial energy of $10 \mathrm{keV}$, an initial cross section of $2 \times 4 \mathrm{~mm}$, and a mass-to-charge ratio value of 1431.5 . A value of $\epsilon_{\text {trans }}=30 \%$ was measured experimentally.

and the CCB were created on the potential array. These electrodes were then assigned experimentally measured potential values $(D F=8010 \mathrm{~V}, C C B=9250 \mathrm{~V}$, $A F=7940 \mathrm{~V}$ ). In this representation, the view is shown from the top of the collision cell. The original collision cell block consists of a hollow stainless steel box containing three regions separated by apertures. The cylindrical GC is inserted via a probe into the middle chamber, adding two additional apertures to the overall cell. The ion beam must pass through two apertures before reaching the GC and then exit through the same 
number of apertures. The ion trajectories in Figure 5 illustrate several potential areas which may contribute to the decrease in the transmission of the precursor ion beam as a function of increasing the potential on the CCB. Based on this model, as the potential of the cell increases, the focal length of the deceleration lens decreases and the dispersion angle of the ion beam increases. The defocusing property of the deceleration lens results in a greater fraction of ions striking the surfaces of the collision cell, as shown in Figure 5, for a collision cell potential of $9250 \mathrm{~V}$. The transmission efficiency predicted, based on the SIMION modeling over the entire range of collision cell potentials, were in sound agreement with the experimentally observed values. Additionally, the SIMION calculations expressed that a smaller ion beam diameter, with respect to the deceleration lens aperture, would result in a smaller dispersion of the ion beam. One approach to attain this criteria is to use an einzel lens prior to the deceleration lens to minimize the ion beam diameter [19], as shown in Figure 2.

Addition of an Einzel Lens Assembly. The einzel lens (EZ) was installed in the field-free region prior to the deceleration lens before the collision cell assembly. An F7. assembly consists of two ground lens elements, before and after a single active lens element. In addition, a grounded tube was placed on the end of the EZ assembly to shield the path of the ions to the collision cell. A comparison of the transmission efficiency of the precursor ions through the original cell, as a function of cell potential, without and with the $\mathrm{EZ}$ on is shown in Figure 4. The EZ has no effect on ion transmission with collision cell block potentials less than $8000 \mathrm{~V}$ ( $\epsilon_{\text {trans }} \geq 90 \%$ ). However, as shown in Figure 4 , in the region from 8000 to $9960 \mathrm{~V}$ applied to the collision cell block, $\epsilon_{\text {trans }}$ is improved by the use of the EZ. Several additional $\mathrm{EZ}$ configurations were tried with a number of active elements, but these did not further enhance the ion transmission. Although there is a reproducible increase in the precursor ion transmission with the EZ in the lower collision energy region, it is still a factor of three less than the transmission observed at higher collision energies.

Modified Collision Cell. Based on the data shown in Figure 4 and the SIMION modeling in Figure 5, it appeared that a large fraction of the precursor ions were striking the surfaces of the collision cell chamber or being improperly refocused in the acceleration region of the cell. To correct these limitations a new CCB was constructed which differs from the original CCB by the removal of the outer two chambers and the elimination of the probe-mounted collision gas cell. The width and the center position of the collision gas cell are the same as in the original unit. The new collision cell assembly width is approximately one-third that of the original cell thus creating space on either side to add additional focusing elements. On the deceleration side of the cell, the ground and active element retained the same spacing as in the original design. Therefore, these lens elements are now $12 \mathrm{~mm}$ further away from the exit slit of MS-1. On the acceleration side of the new cell the ground lens was kept the same distance from the entrance slit of MS-2 as in the original design. An additional focusing element (AF2) was inserted into the space created between this element and the first acceleration lens element (Figure 2b). The SIMION modeling of the ion trajectories through this modified MS/MS interface, consisting of an EZ and new collision cell with two active acceleration lens elements, is shown in Figure 6 at a collision cell block potential of $9210 \mathrm{~V}$. The initial ion beam was focused by the EZ and injected as a very small diameter beam into the deceleration lens. The ion beam was kept tightly focused into the second mass analyzer. The SIMION modeling of the modified MS/MS interface at collision cell block potentials greater than $9900 \mathrm{~V}$, indicated a decrease in precursor ion transmission. This was a result of the inability of the acceleration lens elements to focus the ion beam into MS-2. Based on this model (with the previous noted exception), good ion trans-
MS/MS Interface

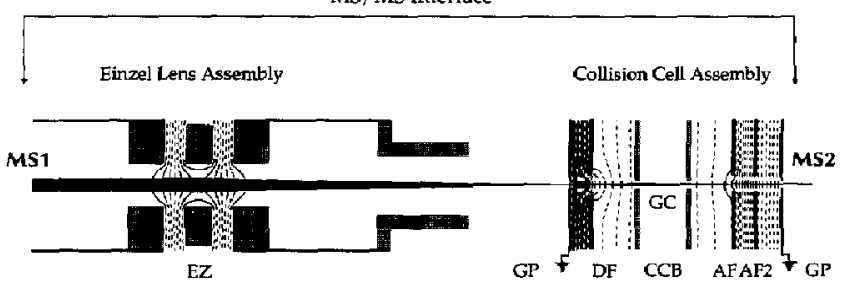

Figure 6. The SIMION of the modified MS/MS interface with a collision cell potential of $9210 \mathrm{~V}$. The electrode elements and their potentials are: $\mathrm{EZ}=4000 \mathrm{~V}$, $\mathrm{DF}=7610 \mathrm{~V}, \mathrm{CCB}=9210 \mathrm{~V}, \mathrm{AF}=7960 \mathrm{~V}$, and $\mathrm{AF} 2=$ $3335 \mathrm{~V}$. All the potentials, with the exception of $\mathrm{EZ}$, were determined experimentally from the optimized interface. The experimentally measured value for EZ, $2090 \mathrm{~V}$, is lower than that used in the model because the quadrupole lens provides some initial focusing of the precursor ion beam and these elemenls are not considered in our current SIMION modeling. The principal direction of the precursor in motion is along the positive $x$-axis. Trajectories are plotted for a precursor ion beam with an initial energy of $10000 \mathrm{eV}$, a mass-tocharge ratio value of 1431.5 , and an initial cross section of $2 \times 4 \mathrm{~mm}$. The value of $\epsilon_{\text {trans }}=91 \%$ was obtained experimentally. 
mission and focusing properties are predicted over a broad range of ion (initial and final) kinetic energies with this interface.

\section{Precursor Ion Transmission with Modified Interface}

The ion transmission characteristics of the modified MS/MS interface were evaluated as a function of the active focusing elements within this region and as a function of the collision cell potential (Figure 7). When the accelerating potential of MS-1 was set to $10,000 \mathrm{~V}$, the ion transmission, as a function of the increasing collision cell block potential from 0 to $8000 \mathrm{~V}$, decreased with the $\mathrm{EZ}$, and second acceleration focusing element off (open boxes, Figure 7a). If the EZ is activated (solid boxes, Figure 7a) the transmission characteristics of the new cell improve in this region. This
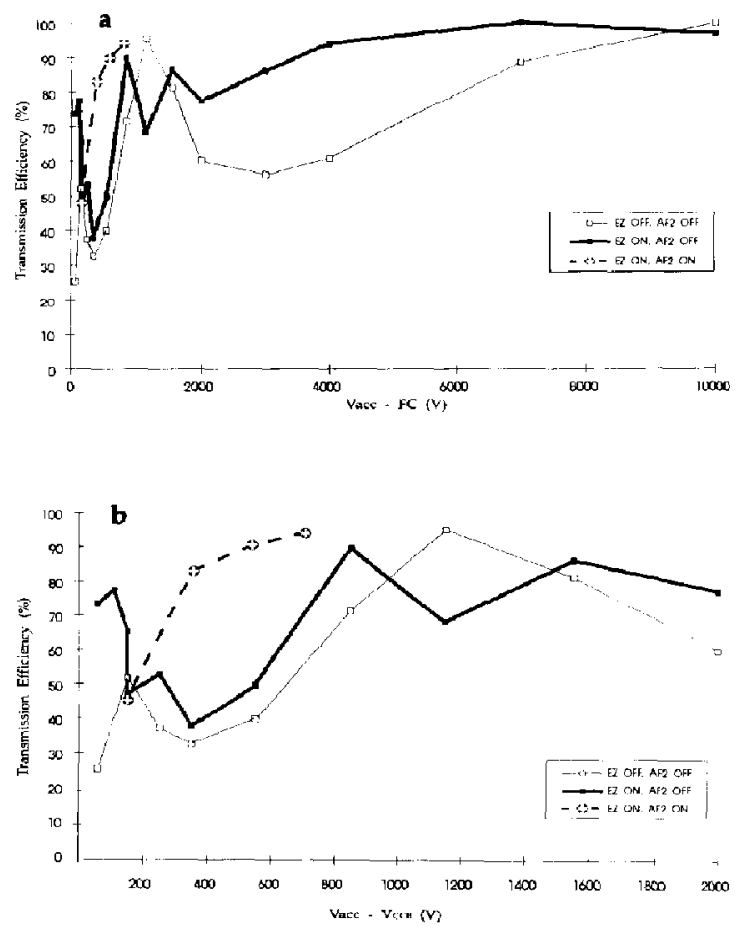

Figure 7. A comparison of the relative precursor ion transmission efficiency through the modified MS/MS interface region as a function of collision energy $\left(V_{\mathrm{acc}}-V_{\mathrm{CCB}}\right)$ and active focusing elements. The transmission efficiencies over the full range from 0 to $10,000 \mathrm{~V}$ applied to the CCB is shown in Figure 7a. The open squares represent the transmission efficiency that is a result only of the modified CCB. An additional improvement in the trans mission efficiency is evident with the activation of the EZ (solid boxes) and the activation of the EZ and second acceleration focusing lens (AF2) (dashed line). The low-collision energy region of this plot is expanded in Figure $7 \mathrm{~b} . V_{\mathrm{acc}}$ and $V_{\mathrm{CCR}}$ are the potentials of MS-1 acceleration and $C C B$, respectively. The reference collision cell potential for the calculation of $\epsilon_{\text {trans }}$ is $0.0 \mathrm{kV}$. difference in transmission may be a result of the change in the focusing point of the ions as a result of moving the ground and deceleration lenses further away from the quadrupole doublet focusing elements immediately after the collector slit of MS-1. Activating the EZ corrects for this change in focusing. Another feature to note is the absence of a dip in the ion transmission in the $\left(V_{a c c}-V_{C C B}\right)$ region from $3000 \mathrm{~V}$ to $4000 \mathrm{~V}$ when the $\mathrm{EZ}$ is on. A dip is routinely observed in this region in the original collision cell.

The combination of the $\mathrm{EZ}$ and the streamlined collision cell block improves precursor ion transmission by approximately a factor of three (i.e., 30\% versus $90 \%$ ) over the original cell at a potential of $9200 \mathrm{~V}$ (solid boxes, Figure 4 versus Figure $7 \mathrm{~b}$ ). However, above collision cell potentials of $9200 \mathrm{~V}$, there is still a dramatic decrease in ion transmission with the new cell block and EZ on (solid boxes, Figure 7b). The transmission in this region can be enhanced by activating the additional acceleration focusing element (AF2). As shown in Figure 70 (dashed line), the transmission is greater than $70 \%$ from 9200 to $9700 \mathrm{~V}$ with this element activated. This additional element improves both precursor and product ion focusing at these cell potentials. A comparison of the measured ion transmission of the original cell (solid boxes, Figure 4) and the modified interface (solid boxes and dashed line, Figure $7 \mathrm{~b}$ ) demonstrate an increase in ion transmission, as a function of the applied cell potential in this region, by a factor of two to four.

\section{Product Ion Spectra with Modified Interface}

The overall efficiency of the modified interface, with all the elements active, was tested with angiotensin I. The CID mass spectrum was acquired at a collision cell block potential of $9150 \mathrm{~V}$ with xenon as the collision gas (Figure 8 ). The tandem mass spectrum is very

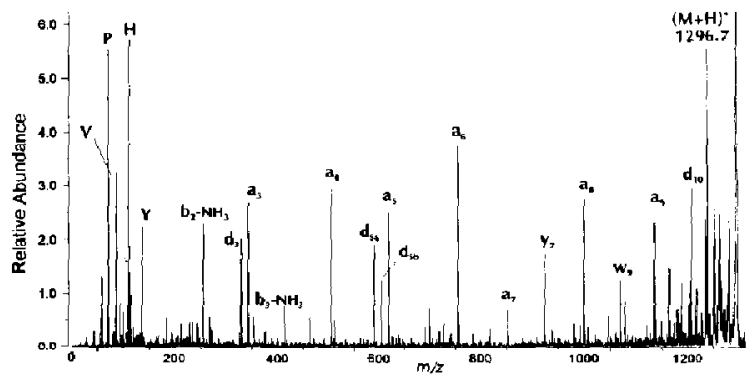

Figure 8. The tandem mass spectrum of angiotensin I (DRVYIHPFHL, $[\mathrm{M}+\mathrm{H}]^{+}$1296.7) obtained using the modified MS/MS interface. The collision energy is $850 \mathrm{eV}$ (lab) or $78 \mathrm{eV}$ (center-of-mass). The precursor ion was attenuated to $25 \%$ with the collision gas, xenon. The apparent CID efficiency is $\epsilon_{a p p a t e n}$ $=7.5 \%$. The product ion distribution is similar to that obtained with the original interface. Furthermore, the $d$ and $w$ ions, which are characteristic of high-energy pathways, are prominent in this spectrum. 
similar to the one acquired with the original interface in Figure $3 \mathrm{~b}$. The differences are: (1) the precursor ion transmission, $\epsilon_{\text {trans }}=92 \%$ in Figure 8 , whereas, $\epsilon_{\text {trans }}$ $=30 \%$ in Figure $3 \mathrm{~b}$ and (2) the product ions in the middle of the mass region in Figure 8 are slightly more intense relative to their immonium ions. This latter effect may be a result of the addition of the second acceleration focusing element. The CID efficiency of angiotensin $I$ in the new interface, $\epsilon_{\text {apparent }}=7.5 \%$, versus that in the original cell, $\epsilon_{\text {apparent }}=6.6 \%$, are similar since the precursor ion transmission is not a factor in the equation for calculating $\epsilon_{\text {apparent }}$. The difference to note is that the overall spectrum quality is better in Figure 8 (i.e., $\epsilon_{\text {overall }}$ is higher, $6.9 \%$ versus $2.0 \%$ ), suggesting that more precursor ions are available for fragmentation. In both tandem mass spectra (Figure $3 \mathrm{~b}$ and Figure 8) $\mathbf{d}$ and $\mathbf{w}$ ions are observed, indicating that the peptide is undergoing high energy fragmentation pathways. These data provide evidence that the modified interface has improved the overall efficiency of the MS/MS process.

Another factor which makes the routine operation at lower collision energies attractive is the reduction in the kinetic energy dispersion of the product ions as a function of the increasing collision cell potential. A comparison of the product ions energy spread as a function of collision energy ( $7000 \mathrm{eV}$ versus $800 \mathrm{eV}$ ) is illustrated in Figure 9. At the lower collision energy (Figure 9b), the lower kinetic energy of the precursor ion, at the point of collision, produces product ions which have a much smaller overall kinetic energy dispersion after acceleration and focusing. As has been pointed out by Biemann and co-workers [14] this smaller energy spread results in a larger fraction of the total product ion spectrum being simultaneously transmitted through the electric sector (i.e., energy filter). This is advantageous if an array detector is used after MS-2 in the MS/MS mode since it would permit the utilization of the maximum array range, independent of the product ion energy. Furthermore, as the collision energy decreases, for example, the potential on the collision cell approaches the accelerating potential of MS-1, the linked scan at constant B/E function approaches a B scan function. The advantage of a B scan for product ion spectra is that it is independent of the precursor ions mass, thereby eliminating the requirement of a unique scan slope for each precursor ion and any errors associated with the resulting linked scan at constant $B / E$ scan function.

In an effort to demonstrate the functionality of this modified MS/MS interface and the effect of enhanced sensitivity at high collision cell block potentials, such as lower collision energies, the tandem mass spectrum of angiotensin I was acquired at a cell potential of 9940 $V$ (i.e., $E_{\mathrm{cm}}=5.5 \mathrm{eV}$ ) (Figure 10a). It has been shown previously that the JEOL HX110/HX110 tandem mass spectrometer is capable of acquiring both high- and low-energy collision spectra of peptides and that there
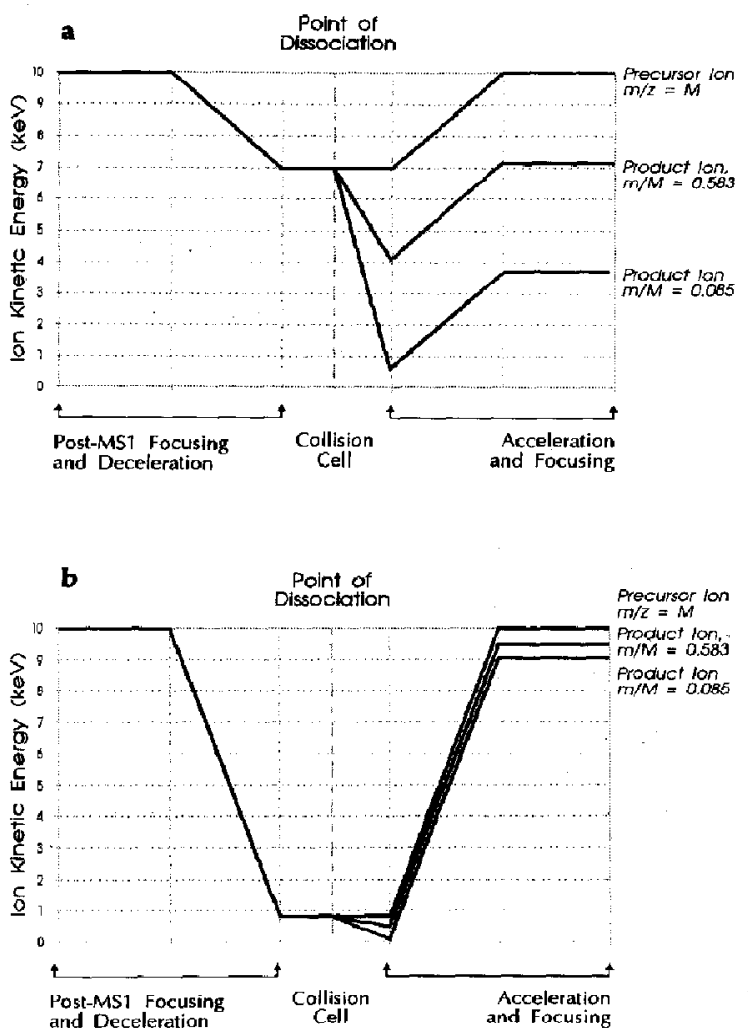

Figure 9. Potential well diagrams for the comparison of the kinetic energy dispersion of the product ions as a function of the collision cell potential. The $y$-axis, on the left side of the diagram, represents the ion source and collision cell potential energy. The $x$-axis is divided into the three regions which define the interface between MS-1 and MS-2. AIl ions have an initial kinctic energy of $10 \mathrm{keV}$ and are decelerated in the post-MS-1 focusing and deceleration region. In order to simplify these diagrams, the point of dissociation of the precursor ion, $M$, is arbitrarily chosen to be in the middle of the collision cell. The product ions, defined as a fraction of the precursor mass, are accelerated and focused with their final energy spread displayed on the right hand $y$ axis. (a) The kinetic energy diagram of a typical CID process with a CCCB potential of $3.0 \mathrm{kV}$. (b) The kinetic energy diagram of a typical CID process with a CCB potential of $9.2 \mathrm{kV}$.

is a transition point from high-energy patterns to lowenergy patterns [15]. As expected, in lower collision energy processes, the $60 \mathrm{eV}$ tandem mass spectrum of angiotensin $I$ exhibits $\mathbf{a}, \mathbf{a}+\mathbf{1}, \mathbf{b}$, etc. The side chain losses such as $d_{5 a}$ and $d_{5 b}$ (Figure 8) are absent. Also observed in this spectrum is a metastable ion of $\mathbf{y}_{9}$ at $\sim m / z$ 1070. It should be noted that in our instrument the precursor ion transmission and product ion focusing with the original interface were so poor as to make the experiment at this collision energy impractical. One important feature of this experiment is, if the accelerating voltage of the instrument is $10,000 \mathrm{~V}$ and an ion 
undergoes a $60 \mathrm{eV}$ collision, the total energy spread for all the product ions is $0.6 \%$. In our instrument the electric sector will transmit all these ions simultaneously. Therefore, a normal magnetic scan at constant electric field potential can be obtained for angiotensin I (Figure 10b). The overall spectral quality and product ion distribution is very similar to that of the spectrum acquired with the conventional linked scan at constant $B / E$ in Figure 10a. This suggests that the product ion spectra could be acquired in this low-energy regime without using the $B / E$ scan function.

\section{Conclusions}

A series of modifications were made to the MS/MS interface in the region between the exit slit of the first mass spectrometer and the entrance slit of the second mass spectrometer on our JEOL HX110/HX110 tandem mass spectrometer. These modifications resulted in an increase in ion transmission over the original design at high $C C B$ potentials of a factor of two to four. These modifications included: (1) addition of an EZ prior to the collision cell to minimize the diameter of the ion beam entering the deceleration lens, (2) minimization of the physical size and path length of
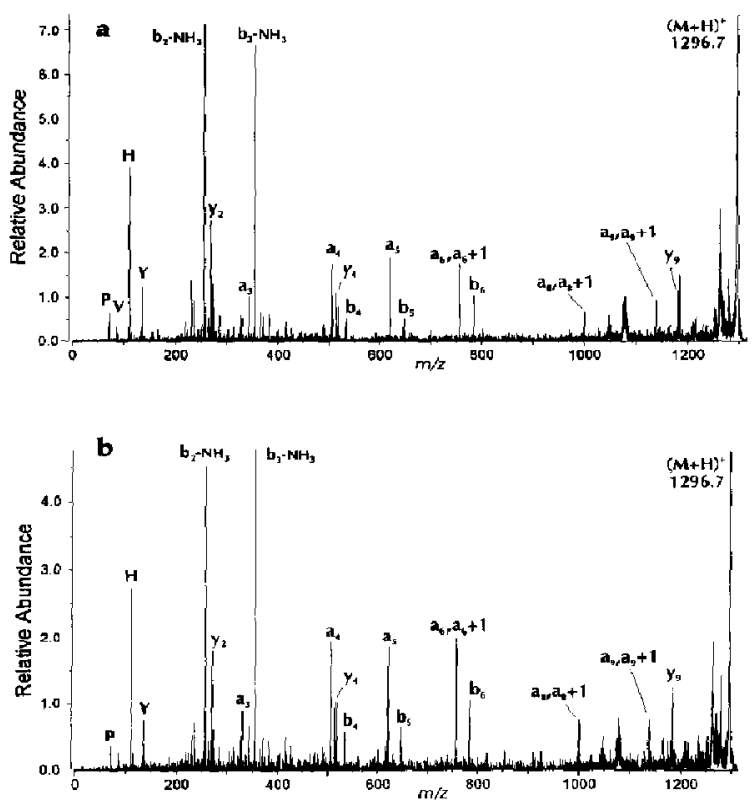

Figure 10. Comparison of the low-energy product ion spectra of angiotensin I obtained with the modified MS/MS interface using (a) a linked scan at constant B/E and (b) a B scan. The collision energy is $60 \mathrm{eV}(\mathrm{lab})$ or $5.5 \mathrm{eV}$ (center-of-mass). Both spectra exhibit very similar distributions of low-energy fragment ions. Furthermore, no $\mathbf{d}$ or $\mathbf{w}$ ions, characteristic of high-energy pathways, were detected. The precursor ion was attenuated to $25 \%$ with xenon as the collision gas. the collision cell block while keeping the gas cell path length constant, (3) reduction of the sources of stray electric fields by removing excess metal, arranging electrical leads so as to minimize their effect on the ions flight path, and shielding the ions flight path whenever practical, and (4) incorporation of a second active element into the acceleration and focusing region of the collision cell.

These modifications to the interface region did not affect the type of fragmentation obtained for peptides as a function of collision cell potential. As expected, $\mathbf{d}$ and $w$ ions were observed at collision energies in the range of $400 \mathrm{eV}$ and greater with xemon as the collision gas, whereas these ions were absent in the collision cell spectra obtained at a collision energy of $60 \mathrm{eV}$. These modifications significantly increased the sensitivity of our tandem sector instrument, enabling good quality tandem mass spectra to be obtained with $60 \mathrm{eV}$ collision energies. The combination of efficient ion transmission at high-collision cell potentials, contubined with existing improvements in ion formation (electrospray) and ion detection $(6 \%$ to $40 \%$ integrating array detectors), should further improve the tandem magnetic sectors' sensitivity and permit existing characterization strategies to be expanded. For example, a real time LC-ESI-MS alternating with LC-ESI-MS/MS during the same high-performance liquid chromatography separation should be practical. If the collision cell is at a very high potential, then only B scans in MS-2 are required, eliminating the need to specify the precursor ion mass and the calculation of the $\mathrm{B} / \mathrm{E}$ scan slope. Therefore, once MS-1 detects an abundant signal in the mass range of interest, the ion is transmitted into the collision cell where it undergoes fragmentation. The product ions are collected at a constant $E$ field by scanning the magnet and integrating up to $40 \%$ of that mass range in a single step. This integrated approach may provide the scan speed and sensitivity to address sequencing problems currently being addressed only by triple quadrupole mass spectrometers [20].

This study was limited to a combination of SIMION and experimentally derived lens potentials for the EZ and modified collision cell lens elements. This approach resulted in improved ion transmission. However, we are not suggesting that these values nor the spatial relationship of all the elements is optimum. This would require comprehensive ion optic modeling of the modifications reported in this paper as well as the standard JEOL dual quadrupole $/(Y$ and $Z)$ lens assembly. We have shown that improvements can be made to the interface region of a tandem magnetic sector instrument which improves overall ion transmission. Furthermore, these data support the hypothesis that increasing the precursor ion transmission will improve the quality of the product ion spectra since the apparent CID efficiency is higher at lower collision energies. Finally, it was hoped that the apparent CID efficiency would increase, that is, a. larger fraction of the product ions would be collected, thereby further 
enhancing the MS/MS sensitivity. This was based on the assumption that the ion optical modifications which improved the precursor ion transmission would also improve the product ions collection. Unfortunately, the enhancement in precursor ion transmission is not mirrored by a significant increase in the relative product ion collection. We are presently addressing the issue of accounting for the majority of the product ion current which is not detected.

\section{Acknowledgments}

We thank Dr. Jason Rouse and Ms. Lisa Housianitis for their critical review of this manuscript.

\section{References}

1. Biemann, K; Martin, S. A. Mass Spectrom. Rev. 1987, 6, 1-76.

2. Hunt, D. F.; Yates, J. R.; Shabanowitz, J.; Winston, S.; Hauer, C. R. Proc. Natl. Acad. Sci. USA 1986, 83, 6233-6237.

3. Sato, K.; Asada, T.; Ishihara, M.; Kunihiro, F,; Kammei, Y.; Kubota, E.; Costello, C. E.; Martin, S.A.; Scoble, H.; Biemann, K. Anal. Chem. 1987, 59, 1652-1659.

4. Spengler, B.; Kirsch, D.; Kaufmann, R. Rapid Commun. Mass Spectrom. 1992, 6, 105.

5. Settineri, C. A.; Medzihradszky, K. F.; Masiarz, F. R.; Burlingame, A. L.; Chu, C.; George-Nascimento, C. Biomed. Environ. Mass Spectrom. 1990, 19, 665.
6. Johnson, R. S.; Biemann, K. Biochemistry 1987, 26, 1209-1214.

7. Papac, D. I.; Oatis, J. E.; Crouch, R. K.; Knapp, D. R. Biochemistry 1993, 32, 5930-5934.

8. Johnson, R. S.; Martin, S. A.; Biemann, K. Int. J. Mass Spectrom. Ion Processes 1988, 86, 137-154.

9. Johnson, R. S.; Martin, S. A.; Biemann, K.; Stults, J. T.; Watson, J. T. Anal. Chem. 1987, 59, 2621-2625.

10. Barber, M.; Bordoli, R. S.; Sedgwick, R. D.; Tyler, A. N. J. C. S. Chem. Comm. 1981, 7, 325-327.

11. Caprioli, R. M. Anal. Chem. 1990, 62, 477A-485A.

12. Fenn, J. B.; Mann, M.; Meng, C. K.; Wong, S. F.; Whitehouse, C. M. Mass Spectrom. Rev. 1990, 9, 37-70.

13. Hillenkamp, F.; Karas, M.; Beavis, R. C.; Chait, B. T. Anal. Chem. 1991, 63, 1193A-1203A.

14. Hill, J. A.; Biller, J. E.; Biemann, K. Int. J. Mass Spectrom. Ion Processes 1991, 111, 1-25.

15. Martin, S. A.; Johnson, R. S.; Costello, C. E.; Biemann, K. In The Analysis of Peptides and Proteins by Mass Spectrometry; McNeal, C. J., Ed.; Wiley: New York, 1988; pp. 135-150.

16. Yost, R. A.; Enke, C. G.; McGilvery, D. C.; Smith, D.; Morrison, J. D. Int. J. Mass Spectrom. Ion Physics 1979, 30, 127-136.

17. Dahl, D. A.; Delmore, J. E.; Appelhans, A. D. Rev. Sci. Instrum. 1990, 61, 607-609.

18. Dahl, D. A.; Delmore, J. E. SIMION PC/PS2 Version 4,0; E66-CS-7233 Rev. 2, April 1988.

19. O'Connor, P. J.; Leroi, G. E.; Allison, J. J. Am. Soc. Mass Spectrom. 1991, 2, 322-335.

20. Hunt, D. F.; Henderson, R. A.; Shabanowitz, J.; Sakaguchi, K.; Michel, H.; Sevilir, N.; Cox, A. L.; Appella, E.; Engelhard, V. H. Science 1992, 255, 1261. 